PROCEEDINGS OF THE

AMERICAN MATHEMATICAL SOCIETY

Volume 132, Number 2, Pages 385-389

S 0002-9939(03)07016-3

Article electronically published on June 10, 2003

\title{
TOPOLOGICALLY MIXING HYPERCYCLIC OPERATORS
}

\author{
GEORGE COSTAKIS AND MARTÍN SAMBARINO
}

(Communicated by Joseph A. Ball)

\begin{abstract}
Let $X$ be a separable Fréchet space. We prove that a linear operator $T: X \rightarrow X$ satisfying a special case of the Hypercyclicity Criterion is topologically mixing, i.e. for any given open sets $U, V$ there exists a positive integer $N$ such that $T^{n}(U) \cap V \neq \emptyset$ for any $n \geq N$. We also characterize those weighted backward shift operators that are topologically mixing.
\end{abstract}

\section{INTRODUCTION}

The notion of hypercyclicity and its consequences has been a concern of operator theorists in the last decade. A continuous linear operator $T$ acting on a (separable) Fréchet space $X$ is said to be hypercyclic provided there is a vector $x \in X$ such that the orbit $\left\{T^{n}(x), n \geq 0\right\}$ is dense in $X$.

It was shown $([\mathrm{A}], \mathrm{B}])$ that any separable Banach space supports such an operator (see also $[\mathrm{BoP}]$ for the case of separable Fréchet spaces). Kitai $[\mathrm{K}]$ and Gethner and Shapiro [GS] provided a useful sufficient condition for an operator to be hypercyclic (see also [G]), namely the Hypercyclicity Criterion. This criterion was refined afterwards by many authors (see $[\mathrm{G}]$ and the references therein). Moreover, Bés and Peris $[\mathrm{BP}$ gave some interesting consequences (in fact, equivalences) of this criterion, such as that $T \oplus T$ is also hypercyclic.

In this work we are concerned with a rather surprising consequence of the Hypercyclicity Criterion: the topologically mixing property. A continuous map $f: Y \rightarrow Y$ on a topological space $Y$ is called topologically mixing if for any given open sets $U, V$ there exists a positive integer $N$ such that $f^{n}(U) \cap V \neq \emptyset$ for any $n \geq N$.

It is well known that $T$ is hypercyclic if and only if given any two open sets $U, V$ there is some positive integer $n$ such that

$$
T^{n}(U) \cap V \neq \emptyset \text {. }
$$

A topologically mixing operator satisfies a much stronger condition: (1) holds for every large $n$. Roughly speaking, the iterates of any open set become well spread throughout the space.

Let us state the above-mentioned criterion in its full generality [BP]:

Hypercyclicity Criterion. Let $X$ be a Fréchet space. An operator $T: X \rightarrow X$ is hypercyclic provided there exist dense sets $D_{1}$ and $D_{2}$ of $X$, an increasing sequence

Received by the editors May 13, 2002 and, in revised form, September 18, 2002.

2000 Mathematics Subject Classification. Primary 47A16, 47B37; Secondary 37B05.

Key words and phrases. Hypercyclic operators, hypercyclicity criterion, topologically mixing. 
of positive integers $\left\{n_{k}\right\}$ and a sequence of maps $S_{n_{k}}: D_{2} \rightarrow X$ such that the following hold:

(1) $T^{n_{k}}(x) \rightarrow_{k} 0$ for $x \in D_{1}$,

(2) $S_{n_{k}}(z) \rightarrow_{k} 0$ for $z \in D_{2}$,

(3) $T^{n_{k}} \circ S_{n_{k}}(z) \rightarrow_{k} z$ for $z \in D_{2}$.

An increasing sequence of positive integers $\left\{n_{k}\right\}$ is syndetic if

$$
\sup _{k}\left\{n_{k+1}-n_{k}\right\}<\infty .
$$

We say that $T$ satisfies the Hypercyclicity Criterion for a syndetic sequence if in the above criterion the sequence $\left\{n_{k}\right\}$ is syndetic. Notice that a large class of hypercyclic operators satisfies the Hypercyclicity Criterion for a syndetic sequence, for instance: $\lambda B$ where $|\lambda|>1$ and $B$ is the backward shift on $\ell^{2}=\ell^{2}(\mathbb{N})$ (the Hilbert space of square summable sequences), and the translation and differentiation operators on the Fréchet space of entire functions $\mathcal{H}(\mathbb{C})$.

Our main result is the following:

Theorem 1.1. Let $T: X \rightarrow X$ be a hypercyclic operator on a Fréchet space. Assume that $T$ satisfies the Hypercyclicity Criterion for a syndetic sequence. Then, $T$ is topologically mixing.

It is not true in general that a hypercyclic operator is topologically mixing (see below). Thus, a natural question is whether a converse of the above theorem holds; in other words, assuming that $T$ satisfies the Hypercyclicity Criterion and is topologically mixing, must $T$ satisfy the Hypercyclicity Criterion for a syndetic sequence? This is true when $T$ is a weighted backward shift as we shall see.

Recall that a unilateral weighted backward shift with weighted sequence $\left\{a_{i}, i \geq\right.$ $1\}$ (bounded and positive) is the (bounded) operator $T: \ell^{2} \rightarrow \ell^{2}$ defined by

$$
T\left(x_{1}, x_{2}, x_{3}, \ldots\right)=\left(a_{1} x_{2}, a_{2} x_{3}, \ldots\right) .
$$

Salas $[\mathrm{S}]$ proved that such an operator is hypercyclic if and only if $\sup _{n} \prod_{i=1}^{n} a_{i}=$ $\infty$. A bilateral weighted backward shift with weighted sequence $\left\{a_{i}, i \in \mathbb{Z}\right\}$ (bounded and positive) is the (bounded) operator $T: \ell^{2}(\mathbb{Z}) \rightarrow \ell^{2}(\mathbb{Z})$ defined by $T\left(e_{i}\right)=$ $a_{i-1} e_{i-1}$ where $\left\{e_{i}, i \in \mathbb{Z}\right\}$ is the canonical basis. Salas, in the paper referred to above, has also characterized the hypercyclic bilateral backward shifts in terms of the sequence $\left\{a_{i}\right\}$.

Theorem 1.2. Let $T$ be a unilateral backward shift with weighted sequence $\left\{a_{i}, i \geq\right.$ $1\}$. Then $T$ is topologically mixing if and only if

$$
\lim _{n} \prod_{i=1}^{n} a_{i}=\infty
$$

Similarly, a bilateral backward shift $T$ with weighted sequence $\left\{a_{i}, i \in \mathbb{Z}\right\}$ is topologically mixing if and only if

$$
\lim _{n \rightarrow+\infty} \prod_{i=1}^{n} a_{i}=\infty \quad \text { and } \quad \lim _{n \rightarrow+\infty} \prod_{i=0}^{n} a_{-i}=0 .
$$

In particular, a weighted backward shift is topologically mixing if and only if satisfies the Hypercyclicity Criterion for a syndetic sequence. 
It is not difficult to find a sequence $\left\{a_{i}, i \geq 1\right\}$ (bounded and positive) where $\sup _{n} \prod_{i=1}^{n} a_{i}=\infty$ holds but (2) is not satisfied, thus providing a hypercyclic (unilateral) weighted backward shift which is not topologically mixing.

In [S] it is also proved that $I+T$ is hypercyclic when $T$ is a unilateral backward shift. León-Saavedra and Montes-Rodríguez [LM] proved that these operators satisfy the Hypercyclicity Criterion. However, it is not clear if the sequence for the Hypercyclicity Criterion can be chosen to be syndetic. We end this section by posing this problem: characterize those unilateral backward shifts so that $I+T$ is topologically mixing.

\section{Proof of Theorem 1.1}

Let $U, V$ be any open sets in $X$. We want to prove that there is some positive integer $N$ such that

$$
T^{n}(U) \cap V \neq \emptyset \forall n \geq N .
$$

Since the sequence $\left\{n_{k}\right\}$ in the Hypercyclicity Criterion is syndetic, there is some positive integer $m$ such that

$$
n_{k+1}-n_{k} \leq m \quad \forall k \geq 0 .
$$

For $i=0,1, \ldots, m$ consider open sets $V_{i}$ such that $T^{i}\left(V_{i}\right)=V$. Choose $x \in U \cap D_{1}$ and take $\epsilon>0$ such that the ball $B(x, \epsilon) \subset U$. Also, for each $i=0,1, \ldots, m$ take $z_{i} \in$ $V_{i} \cap D_{2}$ and we may assume that $\epsilon$ is small enough such that the ball $B\left(z_{i}, 2 \epsilon\right) \subset V_{i}$. In what follows we write $\|x\|=d(x, 0)$ where $d$ is the complete invariant metric of the Fréchet space.

Let $k_{0}$ be large enough such that

$$
\begin{gathered}
\left\|T^{n_{k}}(x)\right\|<\epsilon \quad \forall k \geq k_{0}, \\
\left\|S_{n_{k}}\left(z_{i}\right)\right\|<\epsilon \quad \forall k \geq k_{0}, i=0,1, \ldots, m,
\end{gathered}
$$

and

$$
\left\|T^{n_{k}} \circ S_{n_{k}}\left(z_{i}\right)-z_{i}\right\|<\epsilon \quad \forall k \geq k_{0}, i=0,1, \ldots, m .
$$

Set $N=n_{k_{0}}$ and let $n \geq N$. It follows that there is some $n_{k}$ with $k \geq k_{0}$ and $0 \leq r \leq m$ such that

$$
n=n_{k}+r .
$$

Define $z_{n}=x+S_{n_{k}}\left(z_{r}\right)$. Then $\left\|z_{n}-x\right\|=\left\|S_{n_{k}}\left(z_{r}\right)\right\|<\epsilon$ because of (5) and so $z_{n} \in B(x, \epsilon) \subset U$. On the other hand,

$$
T^{n}\left(z_{n}\right)=T^{r}\left(T^{n_{k}}\left(z_{n}\right)\right)=T^{r}\left(T^{n_{k}}(x)+T^{n_{k}} \circ S_{n_{k}}\left(z_{r}\right)\right) .
$$

Now, the vector $T^{n_{k}}(x)+T^{n_{k}} \circ S_{n_{k}}\left(z_{r}\right)$ is in $V_{r}$ because

$$
\left\|T^{n_{k}}(x)+T^{n_{k}} \circ S_{n_{k}}\left(z_{r}\right)-z_{r}\right\| \leq\left\|T^{n_{k}}(x)\right\|+\left\|T^{n_{k}} \circ S_{n_{k}}\left(z_{r}\right)-z_{r}\right\|<2 \epsilon,
$$

where we used (4) and (6) in the last inequality. Since $T^{r}\left(V_{r}\right)=V$ we conclude that $T^{n}\left(z_{n}\right) \in V$, that is, $T^{n}(U) \cap V \neq \emptyset$. The theorem is proved. 


\section{Proof of Theorem 1.2}

We deal first with unilateral backward shifts. Let us show that if (2) is satisfied, then the unilateral weighted backward shift is topologically mixing. Indeed, take the following dense set in $\ell^{2}$ :

$$
D=\left\{\left\{x_{n}\right\} \in \ell^{2}: x_{n}=0 \text { eventually }\right\} .
$$

The hypercyclicity criterion applies for $D_{1}=D_{2}=D$ and the maps $S_{n}=S^{n}$ where $S: D \rightarrow \ell^{2}$ is defined by $S\left(x_{1}, x_{2}, \ldots\right)=\left(0, \frac{x_{1}}{a_{1}}, \frac{x_{2}}{a_{2}}, \ldots\right)$ and $\left\{n_{k}\right\}=\mathbb{N}$ (notice that the map $S$ may not be well defined either as a map or as a bounded operator with domain $\ell^{2}$ if the sequence $\left\{a_{i}\right\}$ is not bounded away from zero; however it always makes sense when we restrict $S$ to the set $D$ ). Hence, Theorem 1.1 applies and $T$ is topologically mixing.

On the other hand, let us prove that if $T$ is topologically mixing, then (2) holds. Arguing by contradiction, assume that this is not true, that is, $\liminf _{n} \prod_{i=1}^{n} a_{i}<$ $\infty$. In other words, there exist $M>0$ and $n_{k} \rightarrow \infty$ so that

$$
\prod_{i=1}^{n_{k}} a_{i}<M \text { for all } k \text {. }
$$

Consider $e_{1}=(1,0,0, \ldots) \in \ell^{2}$. Let $\epsilon<\frac{1}{2}$ and take $\delta<\frac{1}{2 M}$. Let $U$ be the ball of radius $\delta$ centered at the origin and let $V$ be the ball of radius $\epsilon$ centered at $e_{1}$. Since we are assuming that $T$ is topologically mixing, there is some $m$ so that $T^{n}(U) \cap V \neq \emptyset$ for all $n \geq m$. Take $n_{k}>m$. Thus $T^{n_{k}}(U) \cap V \neq \emptyset$, therefore there is a vector $x=\left\{x_{n}\right\} \in U$ such that $T^{n_{k}}(x) \in V$. Let $x_{n_{k}}$ be the $n_{k}$-component of $x$. It follows that $\left|x_{n_{k}}\right|<\delta$. On the other hand, $T^{n_{k}}(x)=\left(\prod_{i=1}^{n_{k}} a_{i} x_{n_{k}}, \ldots\right)$, and notice that

In particular

$$
\left|\prod_{i=1}^{n_{k}} a_{i} x_{n_{k}}\right|<M \delta<\frac{1}{2} .
$$

$$
\left\|T^{n_{k}}(x)-e_{1}\right\| \geq\left|\prod_{i=1}^{n_{k}} a_{i} x_{n_{k}}-1\right|>\frac{1}{2}>\epsilon,
$$

a contradiction.

Now, we treat the case of bilateral weighted backward shifts. If (표) holds, consider the dense set in $\ell^{2}(\mathbb{Z})$ :

$$
D=\left\{\left\{x_{n}\right\} \in \ell^{2}: x_{n}=0 \text { if }|n|>k \text { for some } k\right\} .
$$

As before, the Hypercyclicity Criterion applies for $D_{1}=D_{2}=D$ and the maps $S_{n}=S^{n}$ where $S: D \rightarrow \ell^{2}(\mathbb{Z})$ is defined by $S\left(e_{i}\right)=\frac{1}{a_{i}} e_{i+1}$ and the sequence $\left\{n_{k}\right\}=\mathbb{N}$. Therefore, Theorem 1.1 applies and $T$ is topologically mixing.

Let us prove that if $T$ is topologically mixing, then (3) holds. We will argue by contradiction. The case $\liminf _{n} \prod_{i=1}^{n} a_{i}<\infty$ leads to a contradiction as we did for the unilateral shift. Therefore, assume that $\lim _{\sup _{n \rightarrow+\infty}} \prod_{i=0}^{n} a_{-i}>0$. Hence, there exist $c>0$ and a sequence $n_{k} \rightarrow \infty$ such that

$$
\prod_{i=0}^{n_{k}} a_{-i}>c>0
$$

Take $0<\epsilon<c$ and choose $\delta$ so that $c(1-\delta)>\epsilon$. Let $V$ be the ball of radius $\epsilon$ centered at the origin and let $U$ be the ball of radius $\delta$ centered at $e_{1}$. Since $T$ is 
topologically mixing, there exists $m$ such that $T^{n}(U) \cap V \neq \emptyset$ for all $n \geq m$. Pick $n_{k}>m$ and let $\left\{x_{n}\right\} \in U$ be so that $T^{n_{k}+1}\left(\left\{x_{n}\right\}\right) \in V$. However, $\left|x_{1}\right|>1-\delta$ and so

$$
\begin{aligned}
\epsilon>\left\|T^{n_{k}+1}\left(\left\{x_{n}\right\}\right)\right\| & \geq\left|\left\langle T^{n_{k}+1}\left(\left\{x_{n}\right\}\right), e_{-n_{k}}\right\rangle\right| \\
& =\left|\prod_{i=0}^{n_{k}} a_{-i} x_{1}\right|>c(1-\delta)>\epsilon,
\end{aligned}
$$

a contradiction.

Furthermore, from the proof we get that a backward shift is topologically mixing if and only if it satisfies the Hypercyclicity Criterion for a syndetic sequence. This concludes the proof of Theorem 1.2

\section{ACKNOWLEDGMENTS}

We wish to thank the referee for many useful comments.

\section{REFERENCES}

[A] Ansari, Shamim I., Existence of hypercyclic operators on topological vector spaces. J. Funct. Anal. 148 (1997), no. 2, 384-390. MR 98h:47028a

[B] Bernal-González, Luis, On hypercyclic operators on Banach spaces. Proc. Amer. Math. Soc. 127 (1999), no. 4, 1003-1010. MR 99f:47010

[BP] Bés, Juan; Peris, Alfredo, Hereditarily hypercyclic operators. J. Funct. Anal. 167 (1999), no. 1, 94-112. MR 2000f:47012

[BoP] Bonet, José; Peris, Alfredo, Hypercyclic operators on non-normable Fréchet spaces. $J$. Funct. Anal. 159 (1998), no. 2, 587-595. MR 99k:47044

[G] Grosse-Erdmann, Karl-Goswin, Universal families and hypercyclic operators, Bull. Amer. Math. Soc. (N.S.) 36 (1999), no. 3, 345-381. MR 2000c:47001

[GS] Gethner, Robert M. and Shapiro, Joel H., Universal vectors for operators on spaces of holomorphic functions. Proc. Amer. Math. Soc. 100 (1987), no. 2, 281-288. MR 88g:47060

[K] Kitai, Carol, "Invariant Closed Sets for Linear Operators", Ph.D. thesis, Univ. of Toronto, 1982 .

[LM] León-Saavedra, Fernando and Montes-Rodríguez, Alfonso, Linear structure of hypercyclic vectors. J. Funct. Anal. 148 (1997), no. 2, 524-545. MR 98h:47028b

[S] Salas, Héctor N., Hypercyclic weighted shifts. Trans. Amer. Math. Soc. 347 (1995), no. 3, 993-1004. MR 95e:47042

Department of Mathematics, University of Maryland, College Park, Maryland 20742 Current address: Vitinis 25 N. Philadelphia, Athens, Greece

E-mail address: geokos@math.umd.edu

Department of Mathematics, University of Maryland, College Park, Maryland 20742 Current address: IMERL, Fac. Ingenieria, University de la República, CC30 Montevideo, Uruguay

E-mail address: samba@fing.edu.uy 